\title{
SOME MAXIMAL NORMAL SUBGROUPS OF THE MODULAR GROUP
}

\author{
by GARETH A. JONES*
}

(Received 10th August 1985)

\section{Introduction}

For each finite group $G$, let $\mathcal{N}_{G}$ denote the set of all normal subgroups of the modular group $\Gamma=P S L_{2}(\mathbb{Z})$ with quotient group isomorphic to $G$; since $\Gamma$ is finitely generated, the number $N_{G}=\left|\mathcal{N}_{G}\right|$ of such subgroups is finite. We shall be mainly concerned with the case where $G$ is the linear fractional group $P S L_{2}(q)$ over the Galois field $G F(q)$, in which case we shall write $\mathcal{N}(q)$ and $N(q)$ for $\mathcal{N}_{G}$ and $N_{G}$; for $q>3$, $P S L_{2}(q)$ is simple, so the elements of $\mathscr{N}(q)$ will be maximal normal subgroups of $\Gamma$.

When $q$ is a prime $p$, there is one obvious element of $\mathcal{N}(p)$ : for each $n \in \mathbb{N}$, the principal congruence subgroup

$$
\Gamma(n)=\{ \pm A \in \Gamma \mid A \equiv \pm I \bmod n\}
$$

of level $n$, is the kernel of the reduction $\bmod n$ from $\Gamma$ to $P S L_{2}\left(\mathbb{Z}_{n}\right)$; this is an epimorphism, so if we take $n$ to be a prime $p$ we find that $\Gamma(p) \in \mathcal{N}(p)$. A natural question is whether there are any other elements of $\mathcal{N}(q)$ for any $q$; it follows from the normal subgroup structure of $P S L_{2}\left(\mathbb{Z}_{n}\right)$ (see [6] for instance) that apart from the single exception $\Gamma(5) \in \mathcal{N}(4)$, arising from the isomorphism $P S L_{2}(5) \cong P S L_{2}(4)$, any such element would be a non-congruence subgroup of $\Gamma$, that is, would contain no $\Gamma(n)$.

In 1936, Philip Hall [2] published an extension of the Möbius inversion formula which allows one to calculate $N_{G}$ provided one knows the subgroup structure and the number of automorphisms of $G$ (indeed, his method also applies to other finitely generated groups besides $\Gamma$ ). Hall concentrated mainly on the groups $G=P S L_{2}(p)$, where $p$ is prime, and showed that $N(p)=\frac{1}{2}(p-c)$ where $c$ is a constant (which he computed) depending on the congruence class of $p \bmod 120$; this result was rediscovered by Sinkov [9], using a different method, in 1969. In particular, for each prime $p \geqq 13$ we have $N(p) \geqq 2$, so that $\mathcal{N}(p)$ contains a non-congruence subgroup (Newman [7] also demonstrated the existence of such subgroups in $\mathscr{N}(p)$ for primes $p \geqq 37$ in 1968).

The techniques used by Newman and Sinkov are specific to quotient groups of type $P S L_{2}$, as are those of Macbeath [5] who proved in 1967 that $\mathcal{N}(q)$ is non-empty for

*This paper forms part of the Proceedings of the conference Groups-St Andrews 1985. 
each prime-power $q \neq 9$, thus giving further examples of maximal normal subgroups of $\Gamma$ which are non-congruence subgroups. The aim of this note is to show how one can use Hall's method to strengthen Macbeath's result by explicitly calculating $N(q)$. For simplicity, we will restrict our attention to the case where $q=2^{e}$; however, the method is quite general, and indeed Martin Downs (private communication) has calculated $N(q)$ for odd $q$.

Theorem. The number $N\left(2^{e}\right)$ of normal subgroups of the modular group with quotient group isomorphic to $P S L_{2}\left(2^{e}\right)$ is

$$
\left.\frac{1}{e} \sum_{f} \mu\left(\frac{e}{f}\right) \grave{\left(2^{f}\right.}-1\right)
$$

thus $N(2)=1$, and $N\left(2^{e}\right)=(1 / e) \sum_{f} \mu(e / f) 2^{f}$ for all $e>1$.

(Here $\mu$ is the Möbius function, and $\sum_{f}$ denotes summation over all positive divisors $f$ of $e$.)

For small $e$ we have the following values:

\begin{tabular}{c|ccccccccccccc}
$e$ & 1 & 2 & 3 & 4 & 5 & 6 & 7 & 8 & 9 & 10 & 11 & 12 & $\ldots$ \\
\hline$N\left(2^{e}\right)$ & 1 & 1 & 2 & 3 & 6 & 9 & 18 & 30 & 56 & 99 & 186 & 335 & $\ldots$
\end{tabular}

The theorem implies that $N\left(2^{e}\right) \geqq 1$ for all $e$, so we have:

Corollary. If $e \geqq 1$ there is a normal subgroup $N \cong \Gamma$ with $\Gamma / N \cong P S L_{2}\left(2^{e}\right)$; if $e=1$ or $e=2$ then $N=\Gamma(2)$ or $N=\Gamma(5)$, but if $e \geqq 3$ each such $N$ is a non-congruence subgroup of $\Gamma$.

\section{Hall's method}

We will briefly outline Hall's method [2], restricting attention to the case of quotients of $\Gamma$; the extension to other finitely generated groups is obvious.

Let $G$ be any finite group; then each epimorphism $\phi: \Gamma \rightarrow G$ determines an element $N=\operatorname{ker} \phi \in \mathscr{N}_{G}$, and every element of $\mathscr{N}_{G}$ arises in this way. Two epimorphisms $\phi$, $\psi: \Gamma \rightarrow G$ have the same kernel if and only if $\psi=\phi \circ \alpha$ for some $\alpha \in$ Aut $G$, so $N_{G}$ is the number of orbits in this action of Aut $G$ on the set of epimorphisms $\phi: \Gamma \rightarrow G$.

Now $\Gamma$ has a presentation

$$
\Gamma=\left\langle X, Y \mid X^{2}=Y^{3}=1\right\rangle
$$

(see [8]), so if $|G|>3$ then epimorphisms $\phi: \Gamma \rightarrow G$ are in one-to-one correspondence with pairs of elements $x=X \phi$ and $y=Y \phi$ of $G$ such that

(i) $x$ and $y$ have orders 2 and 3 respectively,

(ii) $x$ and $y$ generate $G$. 
Let us call $(x, y) \in G \times G$ a modular pair if it satisfies (i), and a modular generating pair (for $G$ ) if it satisfies (i) and (ii). Then $N_{G}$ is the number of orbits of Aut $G$ in its natural action on the set $\mathscr{G}_{G}$ of all modular generating pairs for $G$. Only the identity automorphism can fix such a pair, so Aut $G$ acts semi-regularly on $\mathscr{G}_{G}$; hence

$$
N_{G}=\frac{n_{G}}{\mid \text { Aut } G \mid},
$$

where $n_{G}=\left|\mathscr{G}_{G}\right|$ is the number of modular generating pairs for $G$.

\section{Proof of the theorem}

We now take $G$ to be the group $G_{e}=P S L_{2}(q)$, where $q=2^{e}$. We write $N_{e}$ for $N(q)=N$ etc. Now Aut $G_{e}=P \Gamma L_{2}(q)$ has order $e \omega_{e}$ where $\omega_{e}=q\left(q^{2}-1\right)$ is the order of $G_{e}$.

To calculate $n_{e}=\left|\mathscr{G}_{e}\right|$, let $m_{e}$ be the number of modular pairs in $G_{e}$; clearly $m_{e}=\tau_{e} \theta_{e}$, where $\tau_{e}$ and $\theta_{e}$ are the numbers of elements of orders 2 and 3 in $G_{e}$. Suppose first that $e$ is odd. Then $\tau_{e}=q^{2}-1$ and $\theta_{e}=q^{2}-q$, so

$$
\begin{aligned}
m_{e} & =\left(q^{2}-1\right)\left(q^{2}-q\right) \\
& =(q-1) \omega_{e} .
\end{aligned}
$$

Each modular pair generates a unique subgroup $H$ of $G$, and each subgroup $H$ is generated by $n_{H}$ such pairs, so

$$
m_{e}=\sum_{H \leqq G} n_{H} .
$$

Dickson ([1], Chapter XII) lists the subgroups $H$ of $G_{e}$, and by inspection the only ones which can be generated by a modular pair are the subgroups $H \cong G_{f}=P S L_{2}\left(2^{f}\right)$, where $f$ divides $e$. There are $\left|G_{e}: G_{f}\right|=\omega_{e} / \omega_{f}$ such subgroups for each $f$, and each of them is generated by $n_{f}=n_{G_{f}}$ modular pairs, so (3.2) becomes

$$
m_{e}=\sum_{f} \frac{\omega_{e}}{\omega_{f}} \cdot n_{f}
$$

Combining (3.1) and (3.3), and cancelling $\omega_{e}$, we get

$$
\sum_{f} \frac{n_{f}}{\omega_{f}}=2^{e}-1
$$

Applying the Möbius inversion formula to this, we deduce that

$$
\frac{n_{e}}{\omega_{e}}=\sum_{f} \mu\left(\frac{e}{f}\right)\left(2^{f}-1\right)
$$


In (2.1), we now put $n_{G}=n_{e}$ and $\mid$ Aut $G \mid=e \omega_{e}$, so that (3.5) gives

$$
N_{G}=N_{e}=\frac{n_{e}}{e \omega_{e}}=\frac{1}{e} \sum_{f} \mu\left(\frac{e}{f}\right)\left(2^{f}-1\right) .
$$

If $e>1$ then $\sum_{f} \mu(e / f)=0$, so

$$
N_{G}=\frac{1}{e} \sum_{f} \mu\left(\frac{e}{f}\right) 2^{f}
$$

When $e$ is even, the only changes are that $\theta_{e}$ is now $q^{2}+q$, and that $G_{e}$ has $\omega_{e} / 12$ subgroups $H \cong A_{4}$, each of which can be generated by 24 modular pairs. Thus we must add $2 \omega_{e}$ to the right-hand sides of (3.1) and (3.3). However, these extra terms cancel in (3.4), so the final result is the same as for odd $e$.

\section{Proof of the corollary}

If $\sum_{f} \mu(e / f) 2^{f}=0$ then by taking the negative terms across to the right-hand side we obtain two different binary representations of the same integer, which is absurd. Thus $N\left(2^{e}\right) \neq 0$ so there exists $N \in \mathscr{N}\left(2^{e}\right)$. If $e=1$ or $e=2$ then by inspection $N=\Gamma(2)$ or $N=$ $\Gamma(5)$, so let $e \geqq 3$. If $N \geqq \Gamma(n)$ for some $n$, then $P S L_{2}\left(2^{e}\right)$ is a homomorphic image of $P S L_{2}\left(\mathbb{Z}_{n}\right)$; however, the only non-abelian composition factors of $P S L_{2}\left(\mathbb{Z}_{n}\right)$ are the groups $P S L_{2}(p)$ for primes $p \geqq 5$ dividing $n$ (see [6], [8]), and $P S L_{2}\left(2^{e}\right)$ is not isomorphic to one of these, as can be seen by comparing orders. Thus $N$ is a non-congruence subgroup.

\section{Remarks}

1. Hall's method can be applied to quotient groups $G$ of $\Gamma$ for which the subgroup structure is more complicated than that of $P S L_{2}\left(2^{e}\right)$. Let $\mathscr{S}$ be the set of subgroups $H \leqq G$ which have modular generating pairs (that is, $n_{H}>0$ ). One defines $\mu_{\mathscr{S}}(H)$, for each $H \in \mathscr{S}$, by

$$
\begin{gathered}
\mu_{\mathscr{S}}(G)=1, \\
\sum_{K \geqq H} \mu_{\mathscr{S}}(K)=0 \quad \text { if } \quad H<G
\end{gathered}
$$

(the summation being over all $K \in \mathscr{S}$ containing $H$ ). If $m_{H}$ and $n_{H}$ are the numbers of modular pairs and of modular generating pairs in $H$, then the analogues of (3.2) and (3.5) are

$$
m_{G}=\sum_{H \leqq G} n_{H}
$$

and

$$
n_{G}=\sum_{H \leqq G} \mu_{\mathscr{S}}(H) m_{H}
$$


(again, both summations are restricted to $H \in \mathscr{S}$ ); this last equation can be verified by applying (5.1) and (5.2) to the right-hand side. Knowing the subgroup structure of $G$, one can calculate $\mu_{\mathscr{S}}(H)$ and $m_{H}$ for each $H \in \mathscr{S}$, and hence determine $n_{G}$ from (5.3); then (2.1) gives $N_{G}$. For the general form of Hall's theory, the reader is strongly urged to read [2].

2. The formula for $N\left(2^{e}\right)$ in the theorem also gives the number of irreducible polynomials of degree $e$ over $G F(2)$, or equivalently the number of orbits of length $e$ in the action of the cyclic group $C_{e}$ on its power-set. It would be interesting to find a natural parametrization of the elements of $\mathcal{N}\left(2^{e}\right)$ using these polynomials or orbits.

3. As shown in $[3,4]$, there is a bijection between triangular maps $\mathscr{M}$ on orientable surfaces and conjugacy classes of subgroups $M \leqq \Gamma$; the map $\mathscr{M}$ is regular if and only if $M$ is normal, in which case the orientation-preserving automorphism group Aut ${ }^{+} \mathscr{M}$ is isomorphic to $\Gamma / M$. Thus for any finite group $G, N_{G}$ is the number of regular orientable triangular maps $\mathscr{M}$ with Aut $^{+} \mathscr{M} \cong G$. For instance, the fact that $N(4)=1$ shows that there is just one such map with $\mathrm{Aut}^{+} \mathscr{M} \cong P S L_{2}(4)$; it is, of course, the icosahedron.

Acknowledgement. The author is grateful to the referee for some very helpful comments.

\section{REFERENCES}

1. L. E. Dickson, Linear groups (Teubner, Leipzig, 1901; reprinted Dover, New York, 1958).

2. P. HaLl, The Eulerian functions of a group, Quarterly J. Math. Oxford 7 (1936), 134-151.

3. G. A. JoNEs, Triangular maps and non-congruence subgroups of the modular group, Bull. London Math. Soc. 11 (1979), 117-123.

4. G. A. Jones and D. Singerman, Theory of maps on orientable surfaces, Proc. London Math. Soc. (3) 37 (1978), 273-307.

5. A. M. Macbeath, Generators of the linear fractional groups, Proc. Sympos. Pure Math vol. 12 (Amer. Math. Soc., Providence, R.I., 1967), 14-32.

6. D. L. MCQUILLAN, Classification of normal congruence subgroups of the modular group, Amer. J. Math. 87 (1965), 285-296.

7. M. Newman, Maximal normal subgroups of the modular group, Proc. Amer. Math. Soc. 19 (1968), 1138-1144.

8. M. Newman, Integral matrices (Academic Press, New York, 1972).

9. A. Sinkov, The number of abstract definitions of $\operatorname{LF}(2, p)$ as a quotient group of $(2,3, n)$, J. Algebra 12 (1969), 525-532.

Department of Mathematics

UNIVERSITY OF SOUTHAMPTON

SOUTHAMPTON SO9 5NH 